\title{
Modellierung und Simulation von Batteriespeichern bei der Erbringung von Primärregelleistung
}

\author{
J. Marchgraber, W. Gawlik, C. Alács
}

\begin{abstract}
Die zunehmende Anzahl an über Umrichter angebundenen Erzeugungsanlagen und die gleichzeitige Abnahme der Zahl konventioneller Synchronmaschinen führt zukünftig zu einer Abnahme der vorhandenen Schwungmasse im Netz. Damit wird die Frequenzhaltung in Verbundsystemen schwieriger. Der Erbringung von Regelenergie wird also zukünftig eine noch größere Bedeutung zugesprochen werden. Batteriespeicher eignen sich aufgrund ihres schnellen Regelverhaltens sehr gut, um die Frequenzstabilität zu verbessern und zukünftig als Ersatz für die reduzierte Anzahl an Synchronmaschinen zu dienen. Derzeit besteht im europäischen Verbundsystem ein Markt für die Vorhaltung und Erbringung von Regelreserven (Primär-, Sekundär- und Tertiärregelreserven). Andere Regelleistungsprodukte wie Enhanced Frequency Response (EFR) und Virtuelle Schwungmasse (VSM) sind zwar in Diskussion, aber noch nicht in diesen Markt integriert. Die regulatorischen Anforderungen für speicherbegrenzte Einheiten zur Teilnahme an dem vorhandenen Markt für Primärregelreserven sind relativ strikt, sodass ein wirtschaftlicher Betrieb aufgrund der hohen Investitionskosten oftmals schwierig ist. Im vorliegenden Beitrag wird der Betrieb von Batteriespeichern zur Vorhaltung und Erbringung von Primärregelreserven untersucht und dabei ebenfalls das dazu notwendige Lademanagement abgebildet. Dafür werden verschiedene Nachladestrategien gegenübergestellt und der Einfluss verschiedener Auslegungsgrößen von Batteriespeichern auf die Ergebnisse untersucht. Die Ergebnisse zeigen, dass mithilfe geeigneter Umsetzung des Lademanagements die gegebenenfalls dafür anfallenden Kosten reduziert werden können. Zudem zeigen die Ergebnisse, dass ein Trend zur Auslegung von Batteriespeichern hin zu kleineren Kapazitäten und im Vergleich dazu größeren Leistungen und zudem eine Anpassung der regulatorischen Rahmenbedingungen sinnvoll sind. Die zukünftige Einführung von energieärmeren Regelleistungsprodukten würde einen Trend dahingehend weiter verstärken. Die Anpassung der regulatorischen Rahmenbedingungen zur Erbringung von Primärregelreserven wäre sinnvoll, um einerseits einen diskriminierungsfreien Zugang von Batteriespeichern zum Primärregelenergiemarkt zu ermöglichen und zudem eine geeignete Auslegung von Batteriespeichern für zukünftige Regelleistungsprodukte frühzeitig zu fördern.
\end{abstract}

Schlüsselwörter: Primärregelung; Batteriespeicher; Lademanagement

Modeling the dynamic voltage support of inverter-based generation and storage units.

An increasing amount of inverter-based generating units and the concurrent decrease of conventional synchronous generators leads to an ever decreasing inertia of the electrical grid. This trend causes greater frequency stability issues and therefore raises the significance of regulation reserves in the near future. Battery storages are well suited to improve frequency stability and act as a replacement for the decreasing amount of synchronous generators in the grid. At present, there are markets in the European transmission system for regulation reserves (Frequency Containment Reserve (FCR), automatic Frequency Restoration Reserve (aFRR) and manual Frequency Restoration Reserve (mFRR)) however, no markets exist for additional regulation services, which are currently in discussion (Enhanced Frequency Response (EFR), Virtual Inertia (VI)). Units with limited capacity need to fulfill rather strict regulatory requirements in order to participate on the market for Frequency Containment Reserve (FCR). Considering the high investment costs for battery storages, these requirements aggravate an economic operation of such units. This article investigates the operation of a battery storage providing FCR and the necessary SoC-Management, in order to meet the regulatory requirements. For this study, different SoC-Management strategies and storage parameters are considered. The results show, that an appropriate implementation of the SoC-Management can decrease the balancing costs. The results also indicate that operating battery storages with reduced capacity and increased power and an adaptation of the current regulatory requirements could be sensible. Introducing new regulatory services with minor energy demand would further increase this trend. The adaptation of the current regulatory requirements would allow a non-discriminatory access to the market of FCR for battery storages and facilitate battery storages that are suitable for future regulatory services.

Keywords: frequency containment reserve; battery storage; SoC-management

Eingegangen am 6. November 2018, angenommen am 5. Februar 2019, online publiziert am 11. Februar 2019 (c) The Author(s) 2019

\section{Einleitung}

Aufgrund der sinkenden Preise für Lithium-Ionen-Batterien [13] ist eine zunehmende Installation an stationären Batteriespeichern zu beobachten [12]. Aufgrund ihrer Schnelligkeit sind Batteriespeicher sehr gut zum Einsatz am Regelenergiemarkt geeignet. In Ländern, in denen ein Markt für die Vorhaltung und Erbringung von Primär-
Marchgraber, Jürgen, Institut für Energiesysteme und Elektrische Antriebe, Technische Universität Wien, Gußhausstraße 25/370-1, 1040 Wien, Österreich (E-Mail: juergen.marchgraber@tuwien.ac.at); Gawlik, Wolfgang, Institut für Energiesysteme und Elektrische Antriebe, Technische Universität Wien, Gußhausstraße 25/370-1, 1040 Wien, Österreich (E-Mail: wolfgang.gawlik@tuwien.ac.at); Alács, Christian, Institut für Energiesysteme und Elektrische Antriebe, Technische Universität Wien, Gußhausstraße 25/370-1, 1040 Wien, Österreich

(E-Mail: christian.alacs@tuwien.ac.at) 
regelreserven vorhanden ist, bestehen Geschäftsmodelle, mit denen ein Batteriespeicher Erlöse erzielen kann. Für einen wirtschaftlichen Einsatz von Batteriespeichern sind derzeit aufgrund der derzeit noch hohen Investitionskosten jedoch vielfach weitere Erlösmöglichkeiten nötig, welche eine gleichzeitige Erbringung von anderen Netzdienstleistungen neben Primärregelreserven, erfordern. So lassen sich beispielsweise unter Ausnutzung der Speicherkapazität im verbleibenden Arbeitsbereich der Kapazität durch Arbitragegeschäfte Erlöse erzielen, indem Energie zu Niedrigpreiszeiten gespeichert und zu Hochpreiszeiten wieder eingespeist wird [9]. Aufgrund der derzeitig vorliegenden regulatorischen Rahmenbedingungen ist aber eine gleichzeitige Nutzung des Batteriespeichers zur Erbringung von anderweitigen Netzdienstleistungen nur eingeschränkt möglich. Eine Anpassung der regulatorischen Rahmenbedingungen sowie die Einführung neuer Regelleistungsprodukte würde einen wirtschaftlicheren Einsatz von Batteriespeichern ermöglichen.

Im Projekt BatterieSTABIL [11] werden Möglichkeiten untersucht, neben der Erbringung von Primärregelung andere Netzdienstleistungen anzubieten. Dazu wurde ein Batteriespeicher mit 2,5 MW und 2,2 MWh an das Netz angebunden, mit dessen Hilfe nicht nur die Erbringung von Primärregelung in der Realität untersucht werden kann, sondern auch die Möglichkeit zur Erbringung eventueller zukünftiger Regelleistungsprodukte und der gleichzeitigen Erbringung von Netzdienstleistungen erprobt werden kann.

Im Rahmen dieses Projekts wird mit vorliegender Arbeit auf die Vorhaltung und Erbringung von Primärregelreserven und das dazu nötige Lademanagement eingegangen. Dazu wurde ein Modell zur Simulation dieses Verhaltens erstellt, welches es erlaubt, den Einfluss verschiedener Nachladestrategien in Abhängigkeit von der Auslegung und den Parametern des Batteriespeichers zu untersuchen. Dazu werden in Abschn. 1.1 auf die derzeitig gültigen Rahmenbedingungen eingegangen, in Abschn. 1.2 mögliche Nachladestrategien beschrieben, in Abschn. 2 die Methodik vorgestellt und in Abschn. 3 Ergebnisse beschrieben

\subsection{Regulatorische Rahmenbedingungen}

Der österreichische Übertragungsnetzbetreiber Austrian Power Grid AG (APG) schreibt in seinen aktuellen Präqualifizierungsbedingungen von 2014 [4] diverse Rahmenbedingungen für den Einsatz speicherbegrenzter technischer Einheiten für die Erbringung von Primärregelreserve vor, die auf der damaligen Entwurfsversion des NC LFCR [6] basieren. Die derzeitigen Mindestanforderungen für speicherbegrenzte technische Einheiten in diesen Präqualifizierungsbedingungen sind durch zwei Anforderungen definiert:

* Im "Normal State" ist eine kontinuierliche Primärregelungserbringung zu gewährleisten. Die Frequenz befindet sich im Normal State, wenn sie innerhalb des Bands $(49,95 \ldots 50,05) \mathrm{Hz}$ liegt.

* Wird der "Alert State" erreicht, ist, bei einer Frequenzabweichung von $\pm 200 \mathrm{mHz}$, eine Vollaktivierung von Primärregelreserve zumindest für 30 min. zu liefern. Für Frequenzabweichungen kleiner $200 \mathrm{mHz}$ (d.h. keine Vollaktivierung), ist die Aktivierung entsprechend länger zu erbringen. Die Frequenz erreicht den Alert State, wenn eine der folgenden Bedingungen erfüllt ist.

- $|\Delta f|>100 \mathrm{mHz}$ für mindestens 5 Minuten

- $|\Delta f|>50 \mathrm{mHz}$ für mindestens 15 Minuten

Die zur Auslegung von Batteriespeichern wesentlichste Richtlinie ist die Forderung eines 30-min-Kriteriums. Diese legt fest, dass speicherbegrenzte technische Einheiten im Alert State, welcher im Worst Case durch eine kontinuierliche Frequenzabweichung von mindestens $200 \mathrm{mHz}$ charakterisiert ist, in der Lage sein müssen, für mindestens $30 \mathrm{~min}$. die volle Primärregelleistung erbringen zu können. Die Vorhaltung der entsprechenden Energie schränkt den Arbeitsbereich für eine anderweitige Benützung des Batteriespeichers erheblich ein. Das Batteriespeichersystem muss aufgrund des 30-min-Kriteriums so ausgelegt sein, dass im operativen Betrieb bei einem Übergang in den Alert State eine Vollaktivierung der Primärregelreserve für diese 30 Minuten kontinuierlich möglich ist. Diese Anforderung führt zu einem zulässigen Bereich, in welchem sich der Ladezustand der Batterie befinden muss. Um überhaupt einen solchen Arbeitsbereich für die Erbringung von Primärregelleistung im Normal State zu gewährleisten, ist bei der Auslegung der Batterie die Bedingung $E / P_{P R L}>1 \mathrm{~h}$ zu erfüllen, wobei E die Speicherkapazität und $P_{P R L}$ die angebotene Primärregelleistung bedeutet.

Für die nötige Nachladestrategie bestehen folgende Möglichkeiten:

(a) bilanzgruppeninterne Nachladung

(b) bilanzgruppenübergreifende Nachladung

Die Variante (a) erlaubt eine Nachladung, die an keine zeitlichen Vorgaben hinsichtlich Startzeitpunkt und Zeitdauer gebunden ist, solange sie innerhalb der Bilanzgruppe durch andere Kraftwerke ausgeglichen wird. Für eine dabei entstehende Fehlbilanz fallen Ausgleichsenergiekosten an. Durch die Variante (b) ist der Batteriespeicher selbst Akteur am Strommarkt und hat deshalb die Vorgaben von Fahrplänen einzuhalten [5], wobei durch diese Art Ausgleichsenergiekosten entstehen können. Die Fahrpläne sind an eine 15-minütige kontinuierliche Leistungserbringung gebunden. Ihre Anmeldung hat vom Bilanzgruppenverantwortlichen (BGV) mit einer Vorlaufzeit von $t_{A n m}=15 \mathrm{~min}$. an den Bilanzgruppenkoordinator (BKO) zu erfolgen und ist zu jeder vollen Viertelstunde möglich. Im ungünstigsten Fall findet aufgrund der diskretisierten Auslösezeitpunkte die Auslösung also nach knapp 30 min. statt. Die dabei zu berücksichtigende Diskretisierungszeit $t_{\text {Disk }}$ ist abhängig von der aktuellen Uhrzeit und gibt die Restzeit bis zum nächsten Viertelstundenblock im Fahrplanraster an. Im ungünstigsten Fall beträgt $t_{\text {Disk }}=15$ min. Während der Zeit zwischen Anmeldung und Auslösung eines Fahrplans muss der Batteriespeicher weiter Primärregelleistung erbringen können. Im dazu aufwändigsten Fall kann in der Zeit zwischen Anmeldung und Auslösung $\left(t_{\text {vor }}\right)$ eines Fahrplans eine mittlere Frequenzabweichung von $\pm 50 \mathrm{mHz}$ angenommen werden (vgl. [3]). Diese Frequenzabweichung entspricht exakt der Grenze zwischen Normal State und Alert State und entspricht einem Zustand, welcher vom Batteriespeicher für unbegrenzte Zeit beherrscht werden muss. Beispielhaft ist in Abb. 5 gezeigt, dass solche Fälle tatsächlich im realen Frequenzverlauf auftreten. Das heißt, die vorzuhaltende Energie entspricht $E=t_{\text {vor }} \cdot \frac{50 \mathrm{mHz}}{200 \mathrm{mHz}} \cdot P_{P R L}$, wobei $P_{P R L}$ eine Vollaktivierung der Primärregelreserve bedeutet. Abbildung 1 zeigt den Arbeitsbereich in Abhängigkeit der Speicherkapazität und der angebotenen Primärregelleistung, der sich durch das 30-minKriterium ergibt. Das 30-min-Kriterium ergibt einen Bereich (KB), welcher im Normal State nicht erreicht werden darf. Aufgrund der vorzuhaltenden Energie für die Zeit $t_{\text {vor }}$ zeigt die Abb. 1 außerdem einen Entscheidungsbereich (EB), der durch obige Gleichung definiert ist und der den Arbeitsbereich $(A B)$ des Batteriespeichers weiter einschränkt. Überschreitet der Ladezustand (State of charge, SoC) die Grenzen dieses Entscheidungsbereichs, muss die bilanzgruppenübergreifende Nachladung in die Wege geleitet werden. Dieser Vorgang schließt den Handel und die anschließende Anmeldung des geänderten Fahrplans vom BGV an den BKO mit ein. Die Gesamtdauer für diesen Vorgang bis zur Auslösung des entsprechenden Fahrplans entspricht der Zeit $t_{v o r}$. Unabhängig von der Zeitdauer, die der Handel benötigt, ist rein aus regulatorischer Sicht eine Mindestdauer von 


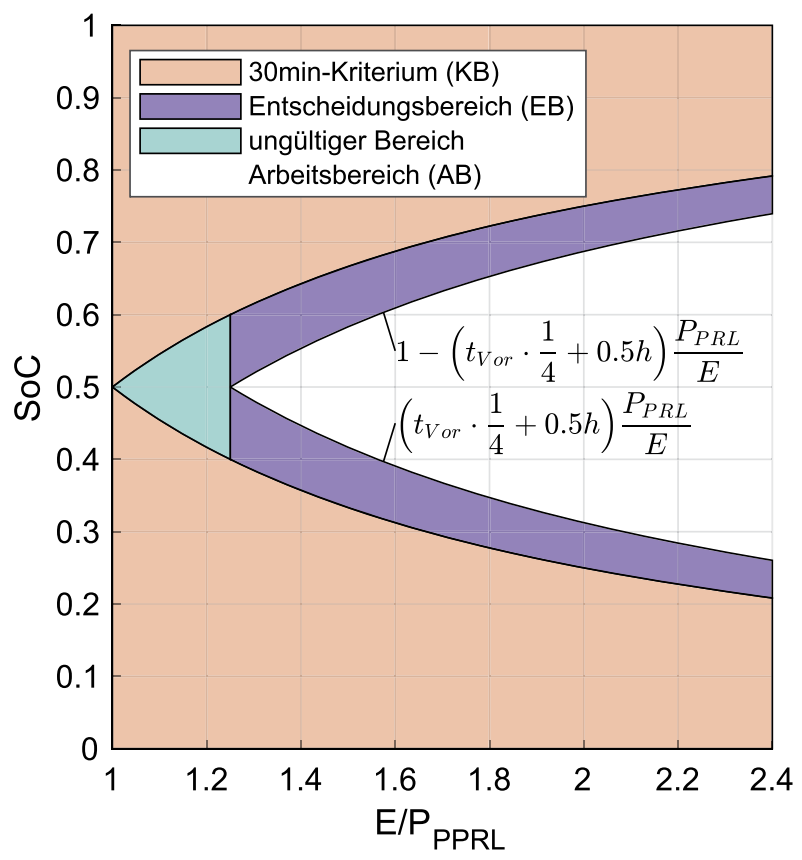

Abb. 1. Speicherverhältnis bei stationären Batteriespeichern unter Annahme einer Vorlaufzeit von $t_{\text {Vor }}=0,5 \mathrm{~h}$

$t_{\text {vor }}=t_{\text {Anm }}+t_{\text {Disk }}=30$ min. für die Auslegung des Entscheidungsbereichs heranzuziehen. Dies bedeutet, dass für diesen Fall ein Kriterium $E / P_{P R L}>1,25 \mathrm{~h}$ bei der Speicherauslegung berücksichtigt werden muss. Der Übergang in den Alert State erfolgt bei einer mittleren Frequenzabweichung von größer $\pm 50 \mathrm{mHz}$ über eine Zeitdauer von 15 min. Dies entspricht dem Abruf einer mittleren Primärregelleistung von $1 / 4 \times P_{P R L}$. Bezüglich der Auslegung der Mindestleistung wird deshalb in den Präqualifizierungsbedingungen [4] eine Überdimensionierung mit $P_{G e s}=P_{F P L}+P_{P R L}>1,25 \cdot P_{P R L}$ vorgeschrieben, um somit durch eine stationäre Arbeitspunktverschiebung die kontinuierliche Primärregelabgabe theoretisch kompensieren zu können. Dabei entspricht $P_{F P L}$ der für die Nachladung verfügbaren Leistung.

\subsection{Implementierung von Nachladestrategien}

Bei der Implementierung der beiden Nachladestrategien besteht die Möglichkeit, sowohl über die Zeit als auch über die Höhe der Leis- tung die nötige Energiemenge zur Nachladung zu erreichen. Für die Variante (a) sind beide Freiheitsgrade frei wählbar. Für Variante (b) ist nur die Leistung frei wählbar, während die Zeit marktbedingt nur in 15-min-Intervallen wählbar ist. Im Anschluss an jede Nachladung wird ein gewisser SoC erreicht. Um die Anzahl an Nachladungen gering zu halten, ist es vorteilhaft, dabei einen gewissen Ziel-SoC anzustreben. Nach [15] ist es günstig, einen Ziel-SoC größer 50\% anzustreben, um die tendenziell unter $50 \mathrm{~Hz}$ liegende Frequenz auszugleichen. Abbildung $2 a$ und $2 b$ zeigen beispielhaft die beiden Nachladestrategien im Vergleich. In Abb. 2a wird dazu nach Strategie (a) bei Überschreitung des in Abb. 1 definierten Bereich des 30-min-Kriteriums (KB) eine Nachladung gestartet, welche mit einer fest vorgegebenen Leistung solange vollzogen wird, bis der Ziel-SoC erreicht wurde.

In Abb. 2b wird nach Strategie (b) bei Überschreitung des in Abb. 1 definierten Entscheidungsbereichs (EB) die Meldung der notwendigen Nachladung an einen Operatorin getätigt. Dieser hat im Entscheidungsbereich, je nach aktueller Uhrzeit, die Zeit $t_{\text {Vor }}-t_{\text {Disk }}-$ $t_{\text {Anm }}$, um eine Nachladung anzumelden. Die anzumeldende Fahrplanleistung entspricht dabei jener Leistung, die zum Zeitpunkt der Fahrplananmeldung notwendig ist, um den Ziel-SoC zu erreichen. Da während der Zeit $t_{\text {Anm }}+t_{\text {Disk }}$ weiter Primärregelleistung erbracht wird, kann der Ziel-SoC zu Ende der Fahrplandurchführung nur näherungsweise erreicht werden.

Wie auch die Ergebnisse in Abschn. 3 zeigen werden, liegt der Wert der Netzfrequenz im langjährigen Mittel tendenziell unter $50 \mathrm{~Hz}$. Bei der Erbringung von Primärregelung mit Batteriespeichern bedeutet dies, dass mehr geladen als entladen werden muss, um den SoC innerhalb des Arbeitsbereichs zu halten. Bilanziell werden deshalb eher Strombezugskosten verursacht. Um während der Erbringung von Primärregelleistung einen SoC innerhalb des Arbeitsbereichs gewährleisten zu können, ist es möglich, den Arbeitsbereich für Arbitragegeschäfte zu nutzen, um die Nachladung zumindest kostenneutral gewährleisten zu können. Abbildung 3 zeigt dazu schematisch die Vorgehensweise. Üblicherweise zeigen sich am Energiemarkt täglich jeweils am Vormittag und am Nachmittag ein Hoch und ein Tief, deren Preisdifferenz genutzt werden kann, um Arbitragegeschäfte durchzuführen. Die Energiemenge, welche im Batteriespeicher zwischengespeichert werden kann, ist durch den Entscheidungsbereich festgelegt.

Im vorliegenden Beitrag wird die Nachladestrategie dahingehend untersucht, wie sich ihre Gestaltung auf den Verlauf des SoC's und
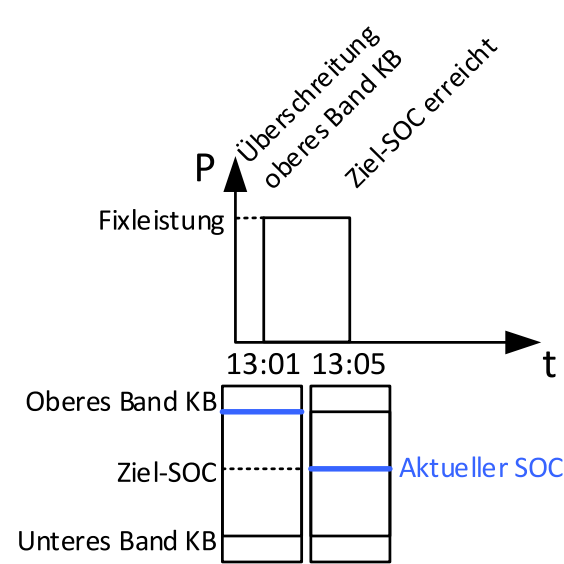

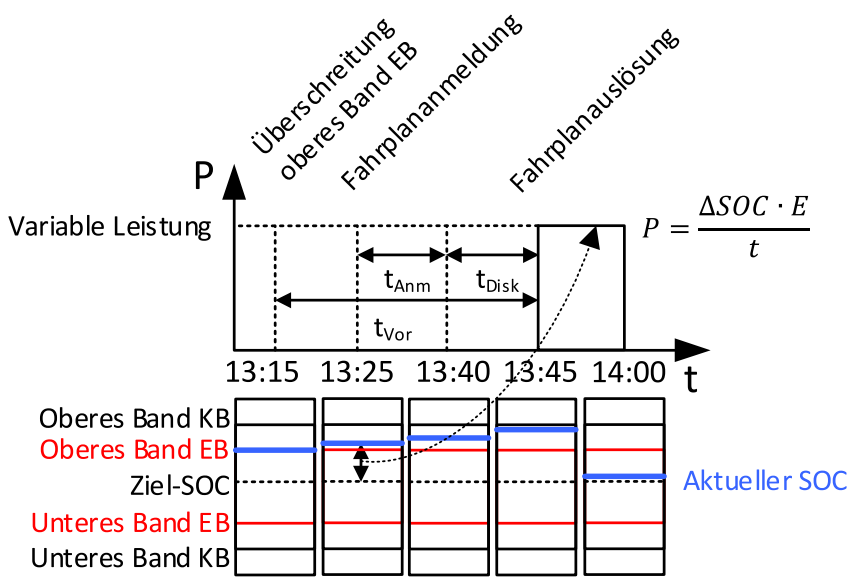

Abb. 2. (a) Nachladestrategie (a) mit fixierter Leistungshöhe bei der Nachladung, (b) Nachladestrategie (b) mit variabel gewählter Leistungshöhe bei der Nachladung 


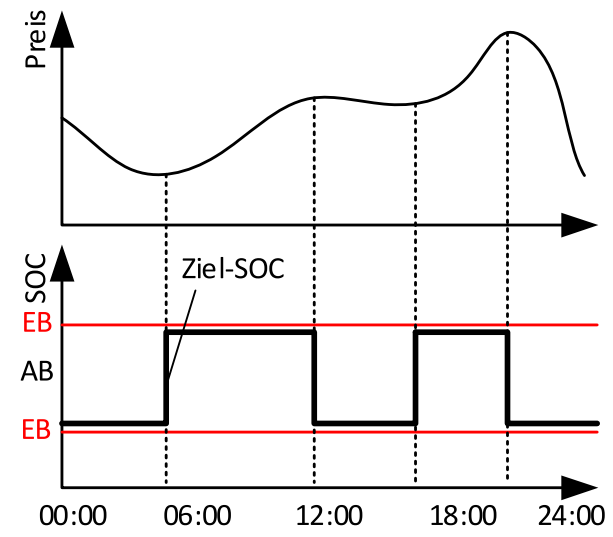

Abb. 3. Variable Anpassung des Ziel-SOC in Abhängigkeit des Energiepreises für Nachladestrategie (b)

die Anzahl an nötigen Nachladungen auf die damit verbundenen Erlöse auswirkt.

\section{Methodik}

Im folgenden Kapitel wird auf die Datengrundlage- und Aufbereitung sowie auf die Auswahl und Modellierung der Szenarien eingegangen.

\subsection{Datengrundlage und Datenaufbereitung}

Um das Verhalten der Primärregelleistungserbringung untersuchen zu können, wurden historische Frequenzdaten aus [1] herangezogen. Simulationen wurden für die gesamte Datenbasis aus dem Jahr 2016 durchgeführt. Gemäß [4] liegt ein Alert State vor, wenn die
Frequenzabweichung für 15 Minuten größer als $\pm 50 \mathrm{mHz}$ oder für 5 Minuten größer als $\pm 100 \mathrm{mHz}$ ist. In Bezug auf Batteriespeicher bedeutet das Vorliegen eines Alert States, dass ein Verlassen des Arbeitsbereichs erlaubt ist. Zudem bleiben nach Vorliegen eines Alert States 2 Stunden Zeit, um mittels Lademanagement wieder in den Arbeitsbereich zurückzukehren. Da der Alert State dementsprechend energetische Auswirkungen auf einen Batteriespeicher hat, wird davon ausgegangen, dass bereits ein arithmetischer Mittelwert der Frequenzabweichung von $\pm 50 \mathrm{mHz}$ für 15 min ausreicht, um diesen zu erreichen. Andernfalls würde eine zeitliche Einordnung, ab wann das 30-min-Kriterium zu gelten beginnt, schwierig durchzuführen sein und gegebenenfalls eine Überdimensionierung von Batteriespeichern zur Folge haben. In Abb. 5 ist ein Ausschnitt der Frequenzdaten dargestellt, welcher die Problematik zur Bestimmung eines Alert State zeigt. Würde alleine die aktuelle Frequenz herangezogen werden, um zu beurteilen, ob ein Alert State vorliegt, würde bereits eine kurze Überschreitung der Grenze von $\pm 50 \mathrm{mHz}$ ausreichen, um diesen zu erreichen. Beim Heranziehen des 15-minMittelwerts ist das Vorliegen einer länger andauernden Frequenzabweichung erforderlich.

Abbildung 4 zeigt eine Übersicht der verwendeten Frequenzdaten in Form eines Wärmebilds. Deutlich zu erkennen sind marktinduzierte Frequenzsprünge zur vollen Stunde bzw. im Fahrplanraster. Diese marktinduzierten Frequenzsprünge ergeben sich dadurch, dass die marktbedingte Reduktion der Leistungseinspeisung eines Kraftwerks A nicht mit einer zeitgleich exakten Ablöse durch die Erhöhung der Leistungseinspeisung eines anderen Kraftwerks B vollzogen werden kann. Neben den eigentlichen Frequenzwerten ist im Wärmebild außerdem gekennzeichnet, zu welchen Zeitpunkten die Bedingung eines Alert State erfüllt ist. Dabei wurden jene Viertelstunden rot markiert, welche ausschlaggebend zur Erreichung des

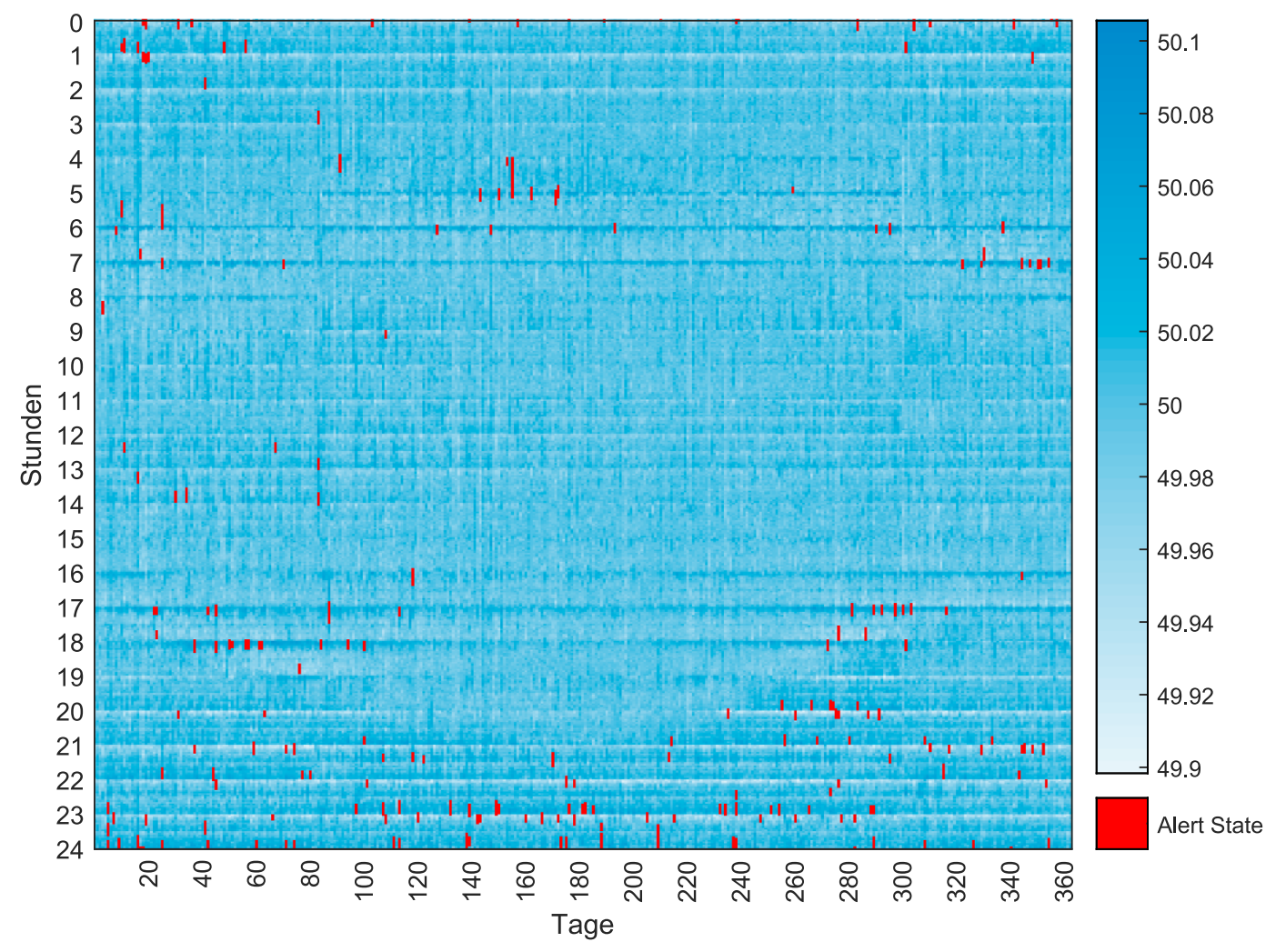

Abb. 4. Datengrundlage der Netzfrequenz 


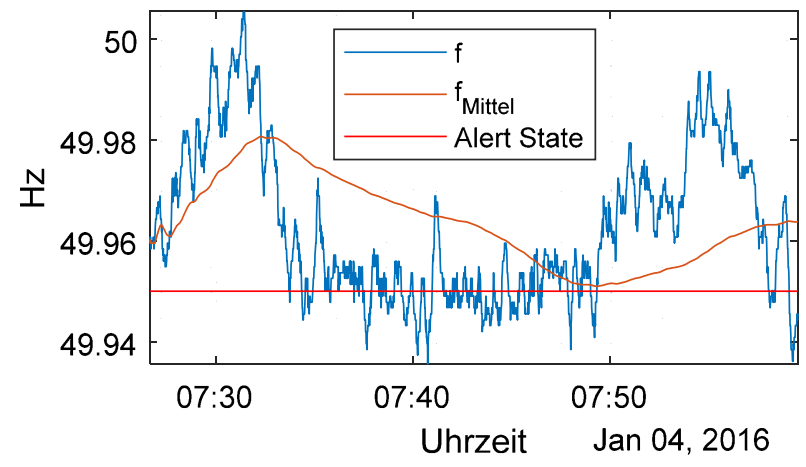

Abb. 5. Ausschnitt aus den Frequenzdaten zur Darstellung eines Frequenzverlaufs nahe der Grenze zum Alert State

Alert State waren. Das heißt, eine Markierung eines Alert State ist zumindest 15 min lang, kann aber auch länger ausfallen. Es zeigt sich, dass Alert States relativ oft und gehäuft am Abend auftreten.

Neben den genannten Frequenzdaten wurde der Jahresdatensatz des Jahres 2016 von Intradaypreisen in 15-min-Auflösung aus [7] zur Simulation der Nachladestrategie in Abb. 3 verwendet. Die Daten wurden so aufbereitet, dass ein entsprechendes Signal für den ZielSoC vorliegt. Dazu wurden in den beiden Tageshälften jedes Tages jeweils das Preisminimum und Preismaximum gesucht und ein Signal gemäß Abb. 3 erzeugt, welches zulässt, dass zwei Arbitragegeschäfte täglich möglich sind. Die dabei vermarktbare Energie hängt ähnlich wie die Bandgrenzen im Arbeitsbereich und Entscheidungsbereich vom E/PPRL-Verhältnis des Speichers ab. Der Ziel-SoC muss innerhalb des Arbeitsbereichs und außerhalb des Entscheidungsbereichs liegen.

\subsection{Modellierung}

Das Verhalten des Batteriespeichers bei Erbringung von Primärregelung sowie das Lademanagement wurden in MATLAB/SIMULINK ${ }^{\circledR}$ modelliert.

Die Primärregelung wurde auf Grundlage von [14] und [4] modelliert und führt zu einer der Frequenzabweichung $\Delta f=f-f_{N}$ proportionalen Leistungsänderung, sobald die Frequenzabweichung das Totband von $\pm 10 \mathrm{mHz}$ überschreitet. Die maximale Primärregelleistung $P_{P R L}$ wird bei einer Frequenzabweichung von $\pm 200 \mathrm{mHz}$ erbracht und ist dort gedeckelt. Dieser Wert muss innerhalb von mindestens $30 \mathrm{~s}$ erreicht werden. Aufgrund von unvermeidbaren Totzeiten im Regelverhalten der Antriebsmaschinen für Synchrongeneratoren ist das dynamische Verhalten für Primärregelanforderungen bei Frequenzabweichungen kleiner $\pm 100 \mathrm{mHz}$ nicht exakt vorgeschrieben, bei entsprechenden Frequenzabweichungen darüber ist aber innerhalb aliquoter Zeitdauern zu $30 \mathrm{~s}$ der entsprechende Arbeitspunkt zu erreichen. Für Batteriespeicher sind üblicherweise keine signifikanten Totzeiten zu erwarten, sodass in dem Fall die dynamischen Anforderungen auch für Frequenzabweichungen kleiner $\pm 100 \mathrm{mHz}$ anwendbar sind. Das bedeutet beispielsweise, dass für Batteriespeicher bei Frequenzabweichungen von $\pm 50 \mathrm{mHz}$ der entsprechende Arbeitspunkt $\pm \frac{1}{4} P_{P R L}$ innerhalb von höchstens 7,5 s erreicht werden muss. Solch eine Formulierung findet sich auch im Entwurf zum Präqualifikationsverfahren für Reserveanbieter in Deutschland [3], in dem angeführt wird, dass die Aktivierung der Primärregelung nicht künstlich verzögert werden darf. Die Einführung eines Totbands zur Einsparung von Primärregelenergie, wie sie z.B. [10] in vorgeschlagen wird und dazu dient, das Lademanagement zu entlasten, gilt damit als nicht zulässig. In [2] werden aber

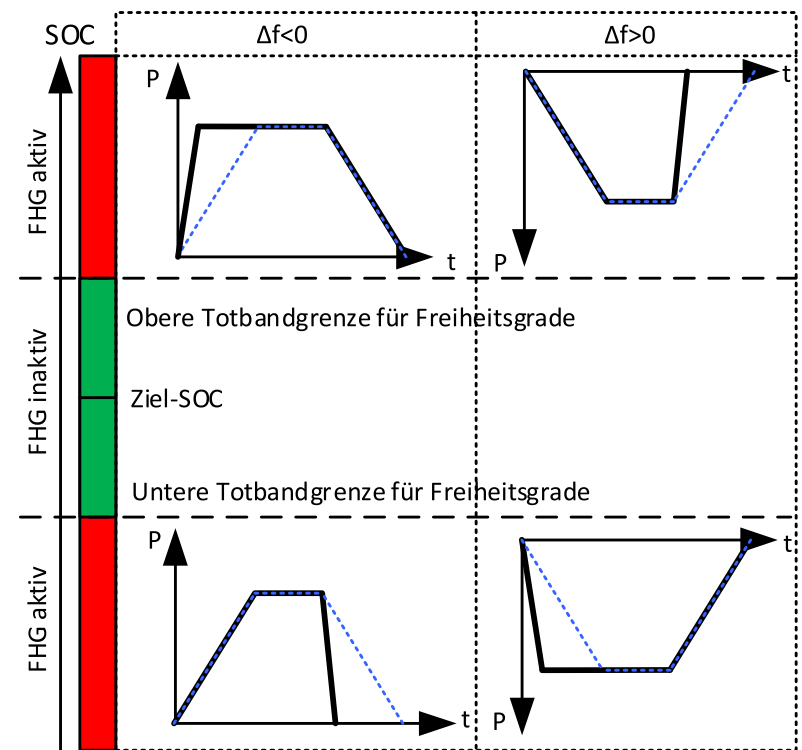

Abb. 6. SOC-abhängige Aktivierung von Freiheitsgraden anhand des Beispiels Anpassung des Gradienten

diverse andere Freiheitsgrade beschrieben, welche speziell für Batteriespeicher vorgeschlagen werden, um das Lademanagement zu entlasten. Zu diesen zählen:

- Übererfüllung: Sofern Primärregelleistung angefordert wird, ist es zulässig, diese um bis zu 20\% überzuerfüllen.

- Nutzung des Totbands: Die lineare Statikkennlinie bei der Erbringung von Primärregelung reicht bis zum Totband. Innerhalb dessen muss keine Leistung erbracht werden. Sofern der linearen Statikkennlinie innerhalb des Totbands aber weiterhin gefolgt wird, ist eine Abgabe von Leistung zulässig.

- Anpassung des Gradienten: Die dynamischen Anforderungen zur Erreichung eines Arbeitspunkts bei vorhandenen Frequenzabweichungen wurden bereits beschrieben. Dort wurde explizit angeführt, dass die angegebenen Anforderungen Mindestanforderungen darstellen, d.h. eine dynamische Übererfüllung ist zulässig

Abbildung 6 zeigt beispielhaft anhand des Freiheitsgrades zur Anpassung des Gradienten, wie die Freiheitsgrade abhängig vom aktuellen SoC aktiviert werden. Eine Aktivierung erfolgt dann, wenn es der schnelleren Erreichung des Ziel-SoC's dienlich ist. Bei allen Freiheitsgraden gilt außerdem die Bedingung, dass diese systemdienlich arbeiten müssen. Das heißt zum Beispiel, dass im Totband bei einer Frequenz größer $50 \mathrm{~Hz}$ keine Leistungsabgabe erfolgen darf und umgekehrt, dass bei einer Frequenz kleiner $50 \mathrm{~Hz}$ kein Leistungsbezug erfolgen darf.

Für das Lademanagement wurden die in Abschn. 1.2 vorgestellten Nachladestrategien modelliert. Während Nachladestrategie (a) eine relativ einfache Umsetzung erlaubt, ist für Nachladestrategie (b) auf einige Besonderheiten zu achten. Als Trigger zur Auslösung eines Fahrplangeschäfts ist neben den Bandgrenzen im Entscheidungsbereich außerdem das Signal für Änderungen im Ziel-SoC zu berücksichtigen. Dabei ist eine Interlocking-Logik erforderlich, die gewährleistet, dass in der Zeit zwischen Triggerereignis eines Fahrplangeschäfts bis zur tatsächlichen Auslösung und dessen Vollendung keine neuen Trigger ausgelöst werden dürfen. Bei langen Vorlaufzeiten hätte dies andernfalls die Folge, dass der Arbeitsbereich verlassen wird oder eine Schwingung im SoC-Verlauf hervorgerufen wird, welche sich zwischen den Bandgrenzen bewegt. Besonders im Alert 
Tab. 1. Übersicht der untersuchten Varianten

\begin{tabular}{lllll}
\hline Zeitkriterium & Vorlaufzeit & Nachlade-strategie & Ziel SOC & Variante \\
\hline $0,5 \mathrm{~h}$ & $0,5 \mathrm{~h}$ & (a) & 0,5 & 1 \\
$0,5 \mathrm{~h}$ & $0,5 \mathrm{~h}$ & (b) & 0,5 & 2 \\
$0,5 \mathrm{~h}$ & $0,5 \mathrm{~h}$ & (b) & variabel & 3 \\
$0,25 \mathrm{~h}$ & $0,5 \mathrm{~h}$ & (a) & 0,5 & 4 \\
$0,25 \mathrm{~h}$ & $0,5 \mathrm{~h}$ & (b) & 0,5 & 5 \\
$0,25 \mathrm{~h}$ & $0,5 \mathrm{~h}$ & (b) & variabel & 6 \\
\hline
\end{tabular}

State und bei niedrigen E/PPRL-Verhältnissen hätte dies negative Auswirkung auf den SoC

Die Simulation wurde mit MATLAB R2018a und einer fixen Schrittweite von $1 \mathrm{~s}$ durchgeführt. Die Variation von Parametern wie z.B. dem E/PPRL-Verhältnis wurde durch parallele Simulation mithilfe von parsim durchgeführt.

\subsection{Szenarienwahl}

Auf Basis der beiden in Abschn. 1.2 vorgestellten Nachladestrategien wurden 6 Varianten, welche in Tabelle 1 zusammengefasst sind, zur Untersuchung ausgewählt und simuliert. Das 30-min-Kriterium ist gemäß [8] derzeit Gegenstand einer Diskussionen hinsichtlich einer möglichen Reduktion auf 15 min., um einen diskriminierungsfreien Zugang von Batteriespeichern zum Primärregelmarkt zu gewährleisten. Neben den beiden Nachladestrategien wurde deshalb auch untersucht, welche Auswirkung eine Anpassung des 30-minKriteriums auf die Ergebnisse hat.

Im Gegensatz zum E/PPRL-Verhältnis, welches einen Mindestwert einhalten muss, besteht für das Verhältnis $E / P_{\text {Ges }}$ die Möglichkeit, geringere Werte zu wählen, indem die Nachladeleistung $P_{F P L}$ höher gewählt wird. Da dies einer unnötigen Überdimensionierung der Wechselrichter gleichkommt, werden Batteriespeicher zur Erbringung von Primärregelung üblicherweise so ausgelegt, dass sich ein Verhältnis $E / P_{\text {Ges }}$ von etwa 1 ergibt. Dies ergibt sich aus der in Abschn. 1.1 erwähnten Forderung $P_{G e s}>1,25 \cdot P_{P R L}$, was bei Einhaltung der Mindestforderung $E / P_{P R L}=1,25 \mathrm{~h}\left(t_{V o r}=0,5 \mathrm{~h}\right)$ ein Verhältnis von $E / P_{G e s}=1 \mathrm{~h}$ ergibt. Eine höhere Wahl von $E / P_{P R L}$ würde nur aus dem Grund erfolgen, dass der damit entstehende Arbeitsbereich für andere Zwecke eingesetzt werden kann. Zur Erbringung von Primärregelleistung würde eine größere Kapazität nur unnötige Investitionskosten verursachen. Wird der Arbeitsbereich z.B. für Arbitragegeschäfte genutzt, muss dementsprechend auch die Nachladeleistung erhöht werden, um die verfügbare Energie auch im am Markt angebotenen Viertelstundenintervall liefern zu können. Nach der Strategie in Abb. 2b wäre dafür genau die Leistung erforderlich, um in einem Viertelstundenintervall den SoC vom oberen zum unteren Ziel-SoC führen zu können. Da dies für größere E/PPRLVerhältnisse eine sehr große Leistung $P_{F P L}$ erfordern würde, wurde in der Szenarienwahl ein Mittelweg zur leistungsbezogenen Überdimensionierung gemäß Tabelle 2 angenommen.

\section{Ergebnisse}

Die Szenarien nach Tabelle 1 wurden mithilfe der beschriebenen Datensätze aus Abschn. 2.1 und dem Modell aus 2.2 simuliert. Die zugehörigen Ergebnisse sind in Abb. 7 dargestellt. Dabei ist im linken Teil der Abbildung das Verhalten der 3 Varianten aus Tabelle 1 an den ersten 5 Tagen im Jahr gegenübergestellt. Die Frequenzdaten sind im oberen Teil der Abbildung dargestellt und weisen am dritten Tag einen Alert State auf, welcher ein Verlassen des Arbeitsbereichs rechtfertigt. Es ist ersichtlich, dass Variante 1 ausschließlich bei Überschreitung der Bandgrenzen des Arbeitsbereichs aktiviert
Tab. 2. Maximal verfügbare Leistung für das Lademanagement

\begin{tabular}{ll}
\hline$E / P_{P R L}$ in $\mathrm{h}$ & $P_{F P L}$ \\
\hline 1,3 & $0,3 \cdot P_{P R L}$ \\
1,4 & $0,6 \cdot P_{P R L}$ \\
1,5 & $0,75 \cdot P_{P R L}$ \\
1,6 & $0,85 \cdot P_{P R L}$ \\
1,7 & $0,95 \cdot P_{P R L}$ \\
1,9 & $1,05 \cdot P_{P R L}$ \\
2,0 & $1,15 \cdot P_{P R L}$ \\
2,1 & $1,25 \cdot P_{P R L}$ \\
2,2 & $1,35 \cdot P_{P R L}$ \\
2,3 & $1,45 \cdot P_{P R L}$ \\
2,4 & $1,55 \cdot P_{P R L}$ \\
\hline
\end{tabular}

wird, während Variante 2 aufgrund des engeren Entscheidungsbereichs öfter getriggert wird. In der dritten Variante überlagern sich den Nachladungen, welche durch Überschreitung der Bandgrenzen des Entscheidungsbereichs ausgelöst werden, jene Nachladungen, welche sich durch Arbitragegeschäfte gemäß Abb. 3 ergeben. Im Fall der ersten beiden Varianten wird als Nachladeleistung immer die maximal mögliche Leistung $P_{F P L}$ gewählt, da der Abstand zum Ziel-SoC zum Zeitpunkt der Fahrplanauslösung ausreichend groß ist. Im Fall der Variante 3 wird bei geringeren Abständen zum Ziel-SoC die Leistung aliquot reduziert. Während des Alert States wird bei der Variante 1 aufgrund der sofortigen Nachladung bei Überschreitung des Arbeitsbereichs der SoC innerhalb des Arbeitsbereichs gehalten. In Variante 2 wird die Nachladung noch im Entscheidungsbereich initiiert, während in Variante 3 aufgrund des geringeren Abstands vom Ziel-SoC zur Grenze des Arbeitsbereichs der SoC den Arbeitsbereich verlässt. Im Fall des Alert States ist dies aber zulässig. Der rechte Teil der Abbildung zeigt die zugehörigen Histogramme, welche auf den Simulationsergebnissen aller Tage des Jahres basieren. Im Histogramm des SoC's der drei Varianten im Vergleich zeigt sich von Variante 1 zu Variante 3 eine zunehmende Ausnützung des Arbeitsbereichs. In den Histogrammen der Nachladeleistungen ist zu sehen, dass der Batteriespeicher häufiger geladen als entladen werden muss.

Abbildung 8 zeigt im oberen Teil die erforderlichen Energien für das Lademanagement der sechs Varianten aus Tabelle 1 in Abhängigkeit vom E/PPRL-Verhältnis im Vergleich. Positive Werte bedeuten dabei abgegebene und negative Werte aufgenommene Energie. Eine vergrößerte Leistung $P_{P R L}$ geht aufgrund der linearen Beziehung, welche eine vergrößerte Primärregelleistung auf die Nachladeenergie hat, direkt in die erforderliche Energie für das Lademanagement ein. Die Ergebnisse gelten also gleichermaßen für größere Primärregelleistungen bei entsprechender Multiplikation der Achsenwerte. Gleiches gilt für den unteren Teil der Abbildung.

Für Variante 1 und Variante 2 ist mit zunehmendem E/PPRLVerhältnis eine Abnahme der nötigen Energien für das Lademanagement zu beobachten. Für Variante 3 ergibt sich in Abhängigkeit des E/PPRL-Verhältnisses eine Zunahme, da aufgrund der dynamischen Anpassung des Ziel-SoC's die Abstände zum Entscheidungsbereich öfter eine Nachladung auslösen als im Falle der beiden anderen Varianten. Für Variante 2 und Variante 3 tritt für geringe $E / P_{P R L}$-Verhältnisse eine höhere Energie für das Lademanagement auf, da der verbleibende Arbeitsbereich relativ gering ausfällt. Während eines Ladevorgangs hat die Leistung zur Erbringung von Primärregelung viel größeren Einfluss auf den $\mathrm{SoC}$ als für größere E/PPRL-Verhältnisse, wodurch nach Abschluss einer Nachladung der 
(a) f/alert
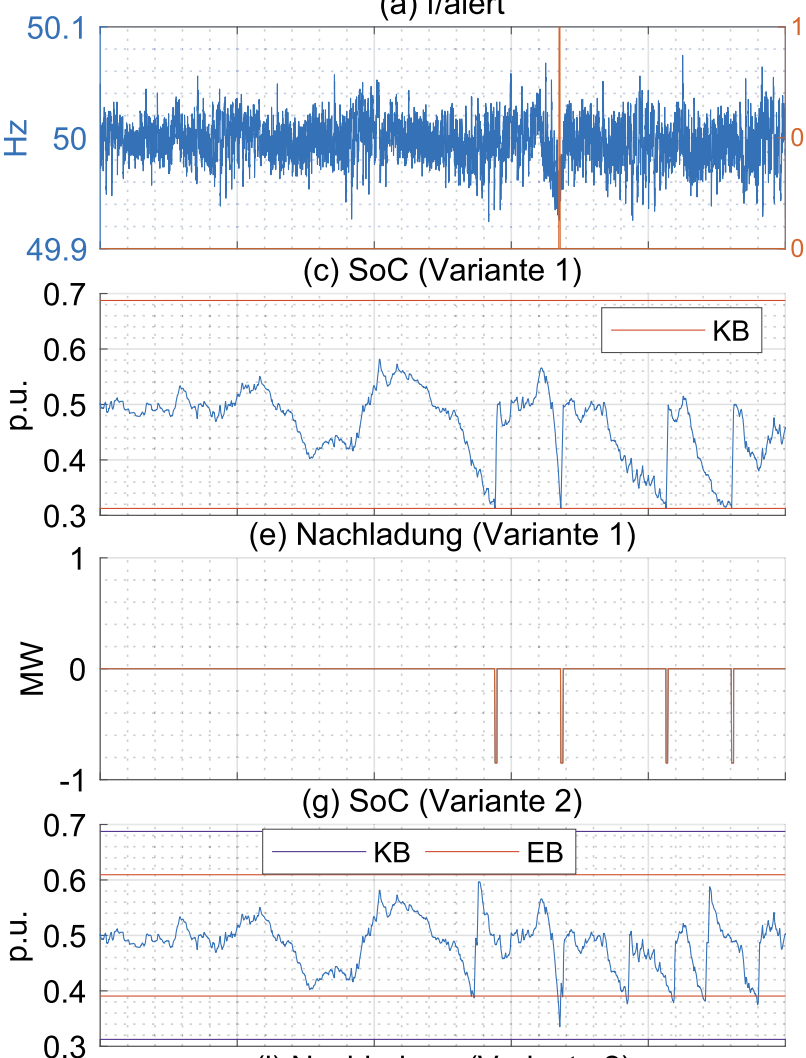

(i) Nachladung (Variante 2)
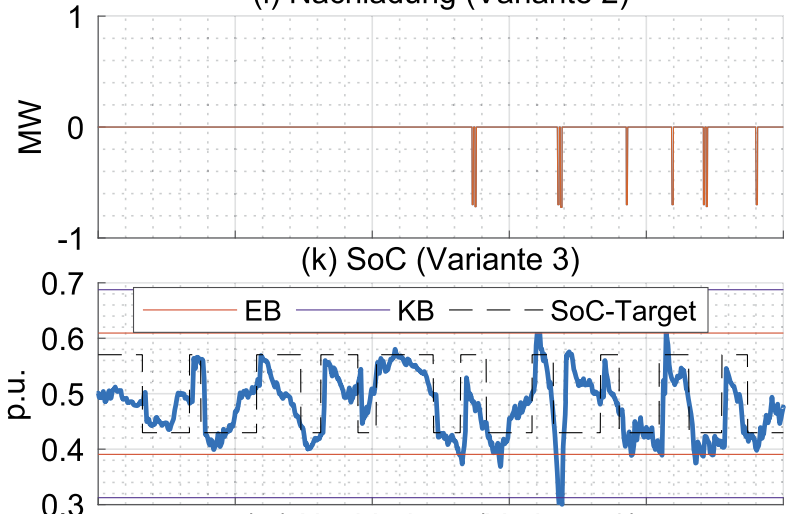

(m) Nachladung (Variante 3)

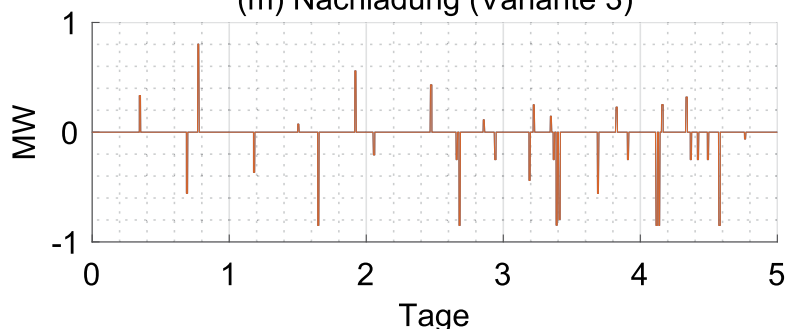

(b) Hist f

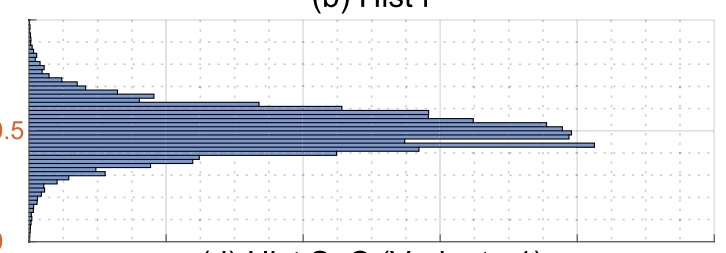

(d) Hist SoC (Variante 1)

0.1

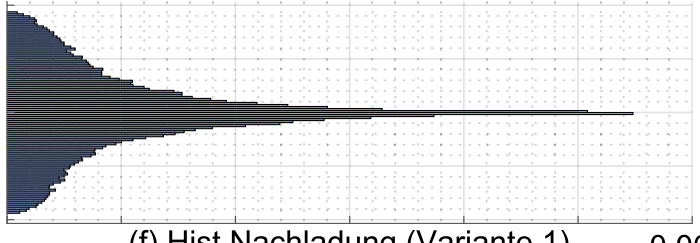

(f) Hist Nachladung (Variante 1) $\quad 0.06$
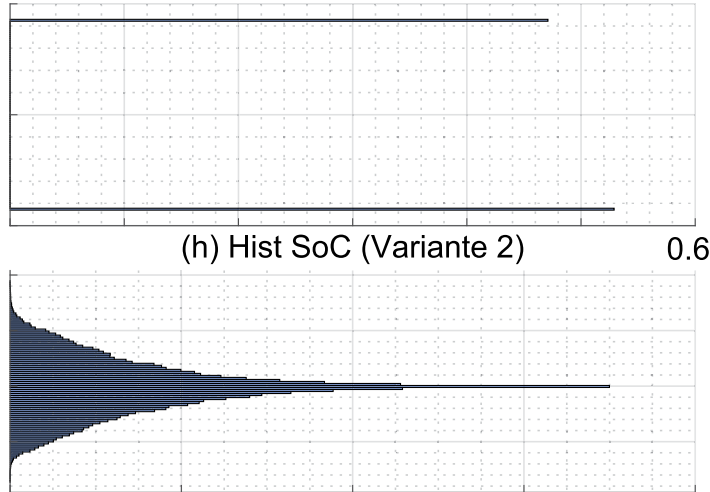

(j) Hist Nachladung (Variante 2)

0.08
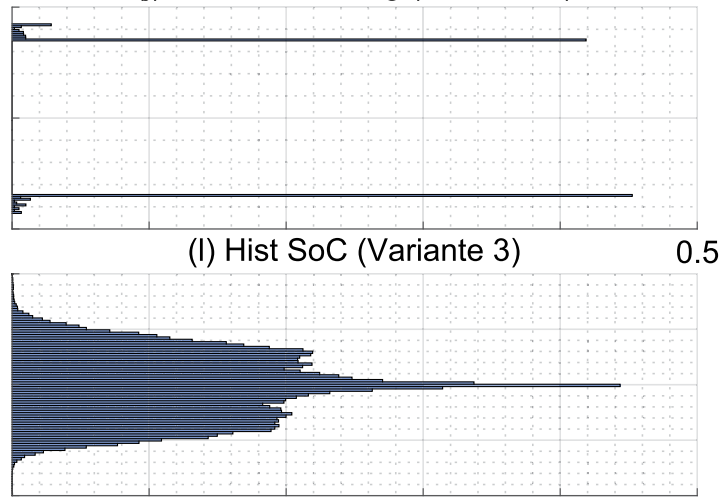

(n) Hist Nachladung (Variante 3)

0.05

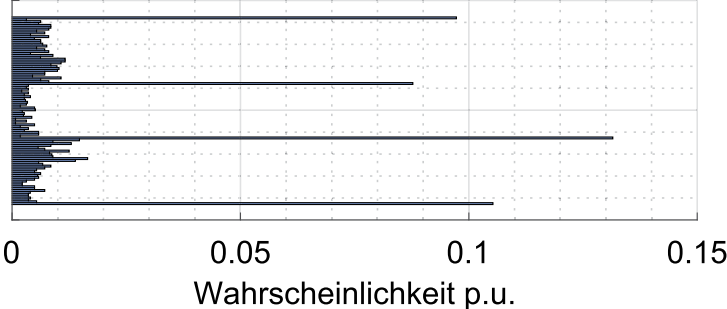

Abb. 7. Simulationsergebnisse der Varianten aus Tabelle 1. Die linke Spalte zeigt den zeitlichen Verlauf der Simulationsergebnisse für die ersten 5 Tage der Datengrundlage aus Abbildung 4, die rechte Spalte zeigt die zugehörigen Histogramme für alle Tage der Datengrundlage

Fall eintreten kann, dass sofort wieder der Entscheidungsbereich erreicht wird.

Der untere Teil von Abb. 8. zeigt die Kosten und Erlöse, welche sich durch das Lademanagement ergeben. Für die durchgeführten Nachladungen wurden die zu diesem Zeitpunkt vorliegenden Intradaypreisen herangezogen. Negative Preise stellen dabei Kosten dar, positive stellen Erlöse dar. Es zeigt sich, dass durch das Nachlademanagement für Variante 1 und Variante 2 Kosten anfallen, wohingegen mittels Variante 3 ab einem E/PPRL-Verhältnis von 1,5 Erlöse erwirtschaftet werden können. Aufgrund der verhältnismäßig geringen Energiemengen sind durch Arbitragegeschäfte auf diese Art zwar keine erheblichen Erträge zu erwarten, jedoch ist es sinnvoll, 

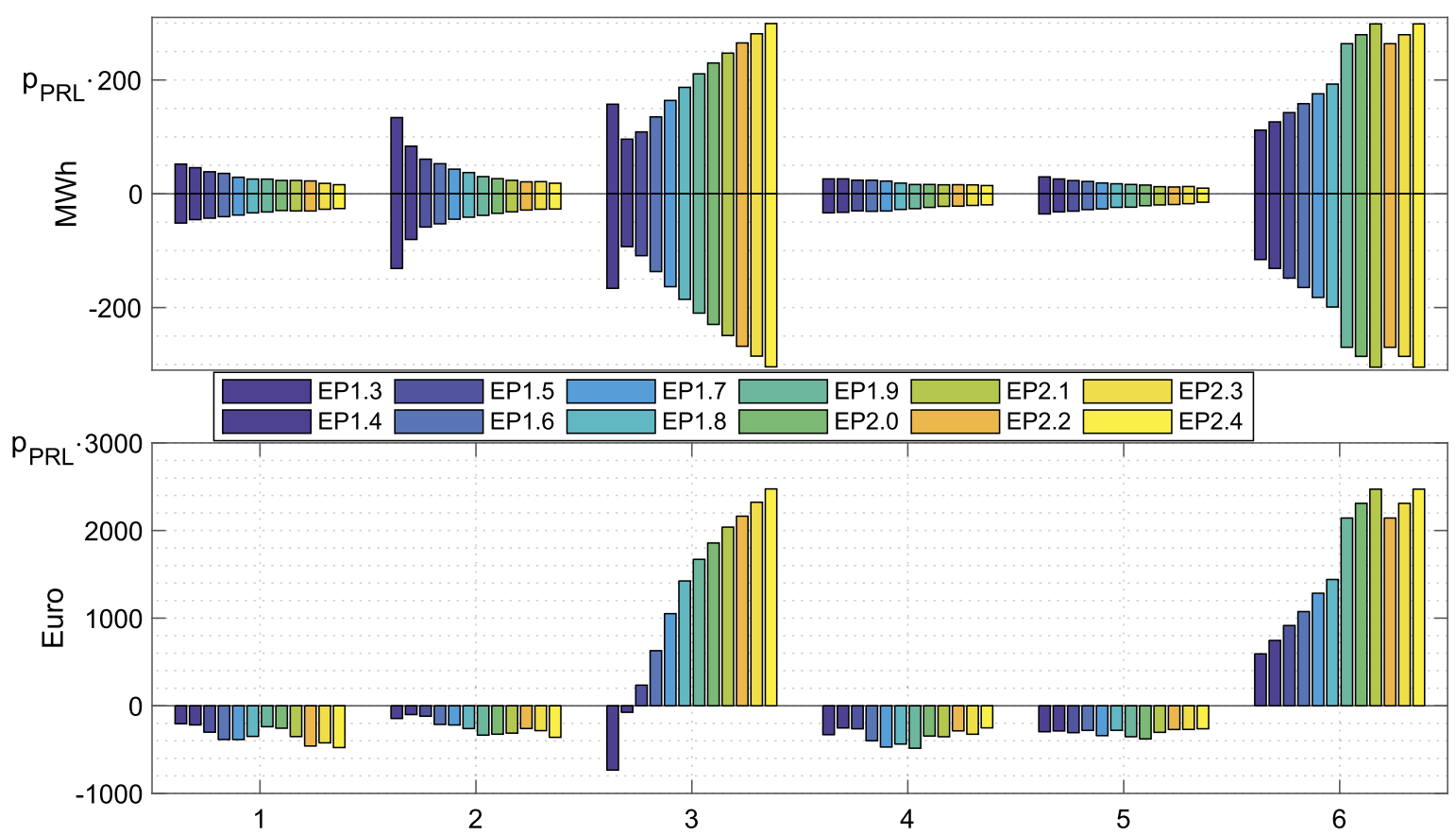

Abb. 8. Vergleich der benötigten Energiemengen und des erzielbaren Erlöses bei der Nachladung unter Verwendung unterschiedlicher Nachladestrategien nach Tabelle 1 in Abhängigkeit des $E / P_{P R L}$-Verhältnisses bei einer Leistung zur Primärregelung von 1 MW. Eine erhöhte Primärregelleistung geht linear in die Ergebnisse ein

das Lademanagement optimiert durchzuführen, sodass zumindest keine Kosten anfallen.

Die Varianten 4 bis 6 entsprechen den Varianten 1 bis 3, jedoch mit dem Unterschied, dass das 30-min-Kriterium auf ein 15-minKriterium geändert wurde. Die erforderliche Energie für das Lademanagement ist im Gegensatz zu den Varianten 1 bis 3 nur geringfügig niedriger. Es ist davon auszugehen, dass bei Vorliegen einer Frequenzabweichung diese tendenziell solange vorliegt, dass auch der vergrößerte Arbeitsbereich nicht dazu beiträgt, das Lademanagement zu reduzieren. Für die Varianten 5 und 6 ist jedoch zu beobachten, dass im Fall von niedrigen E/PPRL-Verhältnissen eine geringere Energie als für Variante 2 und 3 nötig ist. Dies ist darauf zurückzuführen, dass die oben beschriebenen Probleme bei der Nachladung durch den vergrößerten Arbeitsbereich nicht mehr auftreten. Für Variante 6 können zudem unabhängig vom E/PPRLVerhältnis Erlöse durch das Lademanagement erzielt werden.

\section{Zusammenfassung}

Die vorliegende Arbeit beschreibt die Modellierung und Simulation von Batteriespeichern bei der Erbringung von Primärregelleistung. Für das hierfür notwendige Lademanagement wurden mehrere Nachladestrategien untersucht und verglichen. Zudem wurde der Einfluss der Speicherauslegung in Form des E/PPRL-Verhältnisses untersucht. Die Ergebnisse zeigen, dass ohne optimiertes Lademanagement Kosten für das Lademanagement anfallen. Mittels gezielter Anpassungen der Nachladestrategien ist mithilfe von Arbitragegeschäften am Energiemarkt eine Deckung dieser Kosten möglich. Für viele derzeit in Betrieb befindliche Batteriespeicher wird als Geschäftsmodell die Erbringung von Primärregelung herangezogen. Da die regulatorischen Rahmenbedingungen in Form des 30-minKriteriums dafür derzeit eine relativ große Kapazitätsvorhaltung erfordern, ist eine gleichzeitige Nutzung des Batteriespeichers zur Erbringung von anderen Netzdienstleistungen nur sehr eingeschränkt möglich. Eine Anpassung des 30-min-Kriteriums hin zu einem 15min-Kriterium, wie es für konventionelle technische Einheiten verlangt wird, führt zu verringerten Investitionskosten. Ein wirtschaftlicher Betrieb von Batteriespeichern ergibt sich aber oftmals nur durch den Einsatz mehrerer Geschäftsmodelle. Das Anstreben einer moderaten Überdimensionierung der Kapazität bei einem gegebenenfalls zukünftig geforderten 15-min-Kriterium bleibt also bestehen. AuBerdem ist eine Auslegung von Batteriespeichern unter Einhaltung der Bedingung $E / P_{P R L} \ll E / P_{\text {Ges }}$ vorteilhaft. Die Ergebnisse der vorliegenden Arbeit zeigen, dass andernfalls - zumindest für eine Kombination von Primärregelung und gleichzeitiger Durchführung von Arbitragegeschäften - kein Mehrwert durch eine Anpassung des 30-min-Kriteriums entsteht, da der vergrößerte Arbeitsbereich nicht genutzt werden kann. Mit einem derzeit zu erwartendem Trend zu neuen Regelleistungsprodukten, speziell für speicherbegrenzte Einheiten, welche für kürzere Zeitdauern schnell hohe Leistung erfordern, ist ohnehin eine Tendenz bei der Auslegung von Batteriespeichern zu erwarten, welche kleine Kapazitäten, aber hohe Leistungen erfordert. Bei der gleichzeitigen Bereitstellung mehrerer Netzdienstleistungen ist deshalb davon auszugehen, dass dabei vorwiegend energiearme Netzdienstleistungen überlagert werden. Die Aktivierung dieser Regelleistungsprodukte ist nur relativ selten zu erwarten, sodass über längere Zeiträume die Leistungsreserven für andere Zwecke genutzt werden können. Da diese Regelleistungsprodukte im Vergleich zur Primärregelung relativ wenig Vorhaltung von Energie erfordern, entsteht auch für die vorhandene Kapazität ein gröBerer Arbeitsbereich zur anderweitigen Nutzung.

\section{Danksagung}

Open access funding provided by TU Wien (TUW). Diese Arbeit ist Teil des Forschungsprojektes "BatterieSTABIL", gefördert aus Mitteln des Klima- und Energiefonds der österreichischen Bundesregierung. Die Forschungspartner des Projektes sind die Netz NÖ GmbH, die 
Technische Universität Wien, Institut für Energiesysteme und Elektrische Antriebe, Arbeitsgebiet Elektrische Anlagen, und das Austrian Institute of Technology (AIT).

\section{Batterie STABIL

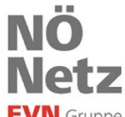

Ein Projekt mit TU Wien und AIT

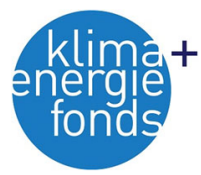

Open Access This article is distributed under the terms of the Creative Commons Attribution 4.0 International License (http://creativecommons.org/ licenses/by/4.0/), which permits unrestricted use, distribution, and reproduction in any medium, provided you give appropriate credit to the original author(s) and the source, provide a link to the Creative Commons license, and indicate if changes were made.

\section{Literatur}

1. 50Hertz (2018): ENTSO-E Netzfrequenz. https://www.50hertz.com/de/Maerkte/ Regelenergie/Regelenergie-Downloadbereich. Zugegriffen: 10.10.2018

2. 50Hertz, Amprion, Tennet, Transnet BW (2014): Eckpunkte und Freiheitsgrade bei Erbringung von Primärregelleistung. https://www.regelleistung.net/ext/download/ eckpunktePRL. Zugegriffen: 15.10 .2018

3. $50 \mathrm{Hertz}$, Amprion, Tennet, Transnet BW (2018) Präqualifikationsverfahren für Regelreservenanbieter (FCR, aFRR, mFRR) in Deutschland ("PQ-Bedingungen"). https://www. regelleistung.net/ext/download/PQ_Bedingungen_FCR_aFRR_mFRR. Zugegriffen: 04.02.2019

\section{Autoren}

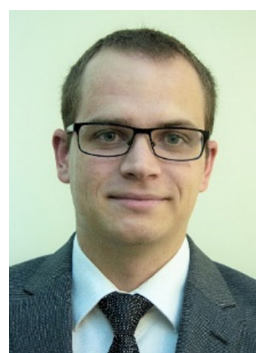

\section{Jürgen Marchgraber}

wurde 1988 in Linz, Oberösterreich, geboren. Er hat das Masterstudium Energietechnik an der Technischen Universität Wien im März 2014 abgeschlossen. Seit 2014 arbeitet er am Institut für Energiesysteme und Elektrische Antriebe der TU Wien als Universitätsassistent.

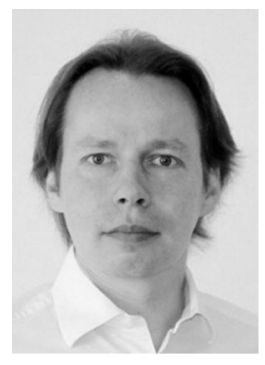

\section{Wolfgang Gawlik}

geboren 1971, hat an der Friedrich-Alexander-Universität (FAU) Erlangen-Nürnberg Elektrotechnik mit Schwerpunkt Energietechnik studiert. Nach Abschluss des Studiums 1997 war er zwei Jahre als Konstrukteur für Turbogeneratoren und stellvertretender Gruppenleiter Neukonstruktion bei der Siemens AG, Generatorenwerk Erfurt tätig. Von 1999 bis 2004 war er Wissenschaftlicher Mitarbeiter
4. AustrianPowerGrid (2018): Erläuterungen Regelreserven. https://www.apg.at/-/ media/3F8C9277B7684F1FBFC1CFF6D933E1AF.pdf. Zugegriffen: 24.09.2018

5. E-Control (2018): Sonstige Marktregeln Strom. Kaptiel 3. Version 5.6. https://www. e-control.at/recht/marktregeln/sonstige-marktregeln-strom. Zugegriffen: 24.09.2018

6. ENTSO-E (2013): Network code on load and frequency control. https://www.entsoe. eu/fileadmin/user_upload/_library/resources/LCFR/130628-NC_LFCR-Issue1.pdf. Zugegriffen: 24.09.2018

7. EPEXSpot (2016): Intraday Preise. Zugegriffen: 15.10 .2018

8. Europäische Kommission (2017): Verordnung (EU) 2017/1485 vom 02.08.2017 zur Festlegung einer Leitlinie für den Übertragungsnetzbetrieb.

9. Hesse, H., Schimpe, M., Kucevic, D., Jossen, A. (2017): Lithium-ion battery storage for the grid — a review of stationary battery storage system design tailored for applications in modern power grids. Energies, 10(12), 2107.

10. Hollinger, R., Diazgranados, L., Sönnichsen, J. (2015): Optimaler Einsatz eines Verbundes von Solar-Batterie-Systemen in der Primärregelleistung. In VDI-Tagung Optimierung in der Energiewirtschaft.

11. Marchgraber, J., Alács, C., Gawlik, W., Kathan, J., Wurm, M., Wailzer, G. (2018): Vitovec W Netzdienstleistungen und Netzstabilisierung - erste Erkenntnisse aus dem Forschungsprojekt "BatterieSTABIL". In Energieinnovation 2018, TU Graz, 14.02.16.02.2018. Graz: TU Graz.

12. Sandia National Laborities (2018): DOE global energy storage database. https:// www.energystorageexchange.org/projects/data_visualization. Zugegriffen: 24.09.2018

13. Statista (2018): Weltweite Preisentwicklung für Lithium-Ionen-Batterien von 2013 bis 2020. https://de.statista.com/statistik/daten/studie/534429/umfrage/weltweitepreise-fuer-lithium-ionen-akkus/. Zugegriffen: 24.09.2018

14. UCTE (2004): A1: Appendix 1: Load-frequency control and performance.

15. Zeh, A., Müller, M., Naumann, M., Hesse, H. C., Jossen, A., Witzmann, R. J. B. (2016): Fundamentals of using battery energy storage systems to provide primary control reserves in Germany. Batteries, 2(3), 29.

und Wissenschaftlicher Assistent am Lehrstuhl für Elektrische Energieversorgung der FAU Erlangen-Nürnberg und promovierte dort 2004. Bis zu seiner Berufung als Universitätsprofessor für Energiesystemtechnik an der Technischen Universität Wien 2011 war er als Senior Key Expert System Dynamics und Projektmanager tätig. Seine Forschungsschwerpunkte umfassen Supergrids, Smart Grids/Microgrids und Elektromobilität, Universal Grids und Software zur Analyse, Planung und stationären und dynamischen Modellierung von Netzen.

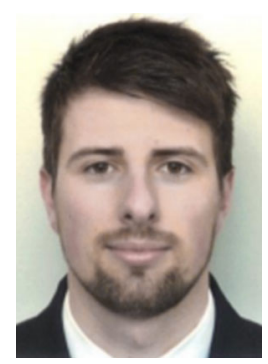

\section{Christian Alács}

wurde 1993 in Wien geboren. Er hat das Masterstudium Energietechnik an der Technischen Universität Wien im März 2017 abgeschlossen. Seit 2017 arbeitet er am Institut für Energiesysteme und Elektrische Antriebe der TU Wien als Projektassistent. 